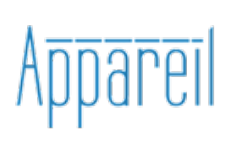

Appareil

$5 \mid 2010$

Identités de la guitare électrique

\title{
La guitare électrique comme moyen de création au sein d'un réseau d'outils compositionnels
}

\section{Santiago Quintans}

\section{(2) OpenEdition}

1 Journals

Édition électronique

URL : http://journals.openedition.org/appareil/1021

DOI : 10.4000/appareil. 1021

ISSN : 2101-0714

\section{Éditeur}

MSH Paris Nord

\section{Référence électronique}

Santiago Quintans, « La guitare électrique comme moyen de création au sein d'un réseau d'outils compositionnels », Appareil [En ligne], 5 | 2010, mis en ligne le 09 juin 2010, consulté le 30 juillet 2020 URL : http://journals.openedition.org/appareil/1021 ; DOI : https://doi.org/10.4000/appareil.1021

Ce document a été généré automatiquement le 30 juillet 2020.

\section{(c) $($ ) $(9)$}

Appareil est mis à disposition selon les termes de la Licence Creative Commons Attribution - Pas d'Utilisation Commerciale - Pas de Modification 4.0 International. 


\title{
La guitare électrique comme moyen de création au sein d'un réseau d'outils compositionnels
}

\author{
Santiago Quintans
}

\section{Introduction}

1 Dans cet article, je voudrais proposer un regard sur la création musicale contemporaine pour la guitare électrique : un regard du point de vue du compositeur/guitariste qui cherche à éclaircir l'identité des outils et des matériaux avec lesquels il travaille pour les organiser d'une façon intelligible, et peut-être, originale et actuelle. Cette démarche suggère trois questions : qu'est-ce qu'une guitare électrique ? (identité), qu'est que l'on peut produire avec elle? (matériaux) et, comment l'organiser dans un tout cohérent? (création).

2 L'identité de la guitare électrique est problématique puisque plurielle. Peut-on parler d'une seule guitare électrique? Comment définir un instrument à l'organologie variable qui joue une multiplicité de rôles dans les musiques et les imaginaires socioculturels mondiaux? Décrire les axes fondamentaux qui sont à l'origine de la guitare est essentiel pour comprendre le processus de mutation qui donne lieu aux multiples identités glocales de cet instrument.

3 Étant donné la multiplicité d'identités de la guitare, il est essentiel de dresser un tableau global des possibilités sonores de cet instrument. Regardant les différentes mutations stylistiques de cet instrument, on peut esquisser le début d'une taxinomie sonore de la guitare électrique, allant du son non amplifié aux sons complexes issus des différentes interactions entre les acteurs d'un réseau complexe (amplificateur, pédales, ordinateur). Cette cartographie sonore de la GE sera le réservoir de matériaux essentiel pour tout travail de création ultérieur.

4 Finalement, organiser un univers sonore tellement riche en interactions ne peut s'accomplir que du point de vue du compositeur contemporain, appliquant à cet 
instrument lié à la musique dite "populaire " une pensée constructive dérivée du monde "savant ». Après avoir dessiné une taxinomie sonore générale de la guitare électrique, il faut donc composer : organiser les matériaux dans un «tout " cohérent. Étant donné que la guitare électrique est elle-même un réseau (corps et amplificateur, pluralité de lieux de contrôle) et qu'elle forme un réseau avec les outils qui l'entourent (les pédales, l'ordinateur, etc.), créer avec elle équivaut à organiser un système d'outils duquel surgiront des événements sonores singuliers. Ici, je m'appuie sur la conception de composition d'Horacio Vaggione: "Composer équivaut à générer des véritables événements singuliers et à les articuler dans des ensembles de plus en plus grands sans perdre le sens de ces singularités. » (Vaggione, 1998)

Cet article a comme point de départ une conception générale de la guitare électrique ; au fur et à mesure que j'avance vers des exemples concrets dans la pratique musicale, je fais des choix marqués par mes intérêts musicaux, et donc des choix plus locaux et personnels (mon activité artistique se situe dans le dialogue entre deux domaines : d'un côté, le travail de musicien interprète de jazz, et d'un autre côté mon activité de compositeur de musique contemporaine). Malgré cette personnalisation de ma recherche, ma proposition, serait applicable à n'importe quelle esthétique ou projet artistique: elle consiste à voir la guitare électrique comme une multiplicité particulière, glocale et à penser la composition comme l'organisation d'un espace d'émergence qui lui serait propre.

\section{Identité(s)}

\section{1. Organologie polarisée}

\section{La guitare électrique est elle-même un réseau}

7 Malgré la multiplicité de formes physiques dans lesquelles l'objet " guitare électrique » s'est incarné depuis sa gestation au début $\mathrm{du} \mathrm{xx}^{\mathrm{e}}$ siècle, la morphologie de la guitare électrique garde une constante : la scission entre la source génératrice de son (le geste instrumental créateur d'énergie) et le lieu de production du son, ce qui implique une multiplication de lieux de contrôle. Autrement dit, la guitare électrique n'est pas un instrument qu'avec un amplificateur. Elle est le générateur d'un « objet » sonore qui va être modelé de plusieurs façons jusqu'à émerger en tant que son. Cette séparation radicale entre « objet générateur » de son et lieu de diffusion constitue un parcours où les « lieux » de manipulation se multiplient (son acoustique, choix de pickup, contrôle de volume et de "tonalité ", pédales, et finalement contrôle de timbre et de puissance de l'amplificateur) créant un réseau de contrôle qui permet de sculpter le signal sonore d'une manière complexe avant qu'il devienne "son ». Ceci fait de la guitare électrique un instrument radicalement différent de la guitare acoustique: avec un instrument acoustique il y a une continuité entre geste instrumental et son dans le geste et dans le temps; avec la guitare électrique cette continuité est brisée, et la notion de geste instrumental devient une variable modulable, composable selon le contexte et l'intention du créateur.

Détournement créatif du réseau : le début d'une identité polarisée

9 C'est dans le «détournement créatif » de ce réseau de base que la guitare électrique trouve ses identités sonores les plus uniques. "Détournement» parce que l'idée originale du tandem guitare-ampli est tout simplement d'amplifier: de faire le son 
physique plus audible. Dès l'instant où les guitaristes commencent à voir les possibilités de ce réseau et à penser cet instrument en tant que tel, la guitare électrique commence à produire des mondes sonores qui lui sont propres. Les exemples de ce phénomène sont maints, et vont du plus simple, comme l'utilisation de l'amplificateur en tant qu'instrument (pour sculpter le son en adaptant la technique acoustique en conséquence), ce qui serait un renversement de la linéarité originale du réseau (la guitare devient simplement source de signal), jusqu'à des conceptions plus complexes comme la création de masses sonores continues avec pédales ou ordinateur, où le son sortant est un mélange des sons à plusieurs points du réseau (son acoustique + son amplifié + son traité). Je reviendrai sur quelques-unes des ces possibilités plus tard, lors de la discussion sur les différentes déclinaisons sonores de la guitare électrique.

10 Si nous voyons donc la guitare électrique comme un réseau, constatant que les différentes manipulations de ce réseau produisent une pluralité de rendus sonores, on peut donc affirmer que, d'un point de vue organologique, la guitare présente une identité multipolaire. C'est cette identité multipolaire qui a permis à la guitare de se développer dans différents domaines et contextes sonores, technologiques, économiques et sociaux.

\section{2. Origines historiques}

11 La pluralité actuelle de la guitare électrique (multiplicité de formes, sons, contextes) n'est pas le fruit d'un hasard, mais d'une évolution au cours du temps dont les origines remontent à il y a plus de cent ans aux États-Unis.

D'abord, il y a une convergence musicale, dans un sens esthétique et fonctionnel. À partir de la deuxième moitié $\mathrm{du}$ xIx ${ }^{\mathrm{e}}$ siècle, la guitare acoustique effectue un rôle d'accompagnement dans la musique populaire nord-américaine, à côté (ou en substitut) d'instruments comme le piano ou le banjo. Cette guitare, aux cordes en métal, joue d'une manière percussive, perçante. Au début du siècle, la guitare hawaïenne devient très populaire aux États-Unis, et le son du steel guitar (guitare posée sur les cuisses du musicien et jouée avec un slide), popularise un son perçant mais à la fois mélodique, qui imite les inflexions vocales de la voix. Cette imitation est aussi recherchée par les guitaristes de blues, un style qui s'appuie largement sur l'expression vocale et le détail sonore des inflexions de la voix. Ces tendances esthétiques, ainsi que la recherche d'une augmentation de la puissance sonore, créent un territoire mûr pour la naissance d'un instrument qui, avec une sonorité plus perçante que sa version acoustique, va prendre une place prépondérante dans les différents styles de musique qui se perpétuent à l'époque.

13 Du point de vue technologique, l'instant était aussi propice à la naissance de cet instrument. Le Gold Rush accélère le développement des industries de câblerie, grâce à la demande croissante de fermetures métalliques pour les territoires gagnés à l'Ouest ; ces développements influencent directement la création de cordes métalliques pour la guitare acoustique, qui à l'époque était équipée de cordes faites de tripes. Le développement des chemins de fer contribue aussi à la popularisation de la guitare, et certains luthiers comme Martin, commencent à envoyer leurs guitares au-delà des centres urbains de production. L'apparition de l'électricité est sans doute cruciale, et marque un tournant au début $\mathrm{du} \mathrm{xx}^{\mathrm{e}}$ siècle. Les développements qui en découlent, notamment liés à l'industrie de télécommunication (téléphone, amplification, radio, 
etc.) ont posé les bases technologiques indispensables pour le développement d'une guitare amplifiée, électrique. Les développements des industries d'entertainment (d'abord la radio, qui « de-localise » les performances, ensuite la télévision, qui rajoute l'image) aident à la popularisation et à l'internationalisation de la guitare électrique, dans un pays culturellement déjà prêt à accueillir cet instrument. Le développement technologique que je viens de décrire comporte aussi un développement d'une industrie autour de la guitare. L'industrialisation des États-Unis marque le début d'un nouveau modèle économique auquel la guitare, grâce à sa portabilité et sa popularité, s'adapte facilement. La vente par courrier s'installe rapidement grâce à la distribution des chemins de fer et, en conséquence, les luthiers commencent à produire en masse en vue de fournir tout le territoire national. L'apparition de plusieurs compagnies de guitare accélère les innovations concernant l'électrification, grâce à la compétition qui s'installe pour dominer le marché: le principal motif de la création de la Broadcaster de Leo Fender, guitare en bois solide faite de pièces détachables, est de trouver un modèle de guitare qui puisse être produit en série dans une usine. Cette industrie n'a fait que grandir et se multiplier jusqu'à nos jours.

15 La guitare, à partir de l'époque du gold rush (où le piano "victorien » est venu la remplacer comme instrument de «salon»), devient un symbole de classe, et éventuellement, elle devient porteuse d'une certaine idée de mécontentement et protestation dans l'imaginaire populaire américain. Des racines folk de la fin du XIX jusqu'à la dépression des années 1930, chantée avec une guitare par Woody Guthrie, en passant par le développement du blues, la guitare devient une sorte de métaphore, un symbole d'individualité qui se rebelle et qui cherche à s'affirmer. Les liens de la guitare électrique avec le marché et l'industrie du mass media génèrent une pluralité d'imaginaires, allant de la rébellion et l'individualisme exacerbé (Hendrix, Van Halen), jusqu'à la négation de l'individu "performant" en faveur d'une création musicale accessible à tous grâce à la guitare (culture indie). Aujourd'hui, ces différentes déclinaisons de l'imaginaire populaire portées par la guitare, avec ses conséquences esthétiques forment un riche paysage international, que certains musicologues comme Kevin Dawe étudient sous le nom de guitarscapes. Cette effervescence musico-culturelle montre le potentiel symbolique d'un instrument qui se transforme et s'adapte aux différentes cultures dans lesquelles il arrive.

\section{3. Une identité multiaxiale}

16 Les domaines sur lesquels s'appuie le développement de la guitare électrique au début $\mathrm{du} \mathrm{Xx}^{\mathrm{e}}$ siècle vont s'internationaliser et se développer à partir des années 1950 . La palette sonore de la guitare électrique s'élargit en touchant à la plupart des esthétiques populaires $\mathrm{du} \mathrm{xx}^{\mathrm{e}}$ siècle. Elle se développe aussi dans le jazz et, timidement, dans la musique contemporaine. Elle ne reste pas à l'écart des développements technologiques et industriels, mutant constamment au contact avec les technologies environnantes (Gibson numérique, par exemple). L'industrie de la guitare électrique se développe dans de nombreux pays, produisant de multiples déclinaisons inspirées des modèles de Fender et Gibson.

Aujourd'hui, la valeur que l'on donne au rôle esthétique, économique, industriel et social d'une guitare électrique varie énormément selon le contexte local dans lequel on 
se situe et c'est cette capacité à renouveler sa valeur à partir d'un axe de domaines de base (esthétique musicale, technologie, économie, société), qui fait de la guitare un instrument extrêmement riche et actuel.

18 Je voudrais donc parler de l'identité de la guitare électrique comme d'une identité multiaxiale variable, qui prend différentes formes selon le contexte glocal dans lequel elle se situe. De cette façon nous pourrons analyser une guitare électrique particulière en étudiant son contexte et les interactions entre les domaines de base qui la définissent.

\section{Glocalités}

19 Il y a quelques déclinaisons glocales de la guitare électrique qui, de mon point de vue de guitariste/compositeur, méritent être étudiées avec soin. Je fais cette précision parce que, à ce stade là, une analyse de toutes les déclinaisons de la guitare électrique serait impossible. J'ai fait donc un choix de quelques « guitares glocales» qui exemplifient un type de matériau sonore ou approche conceptuelle intéressants pour le développement de mon travail. L'analyse multiaxiale/glocale sera donc limitée à l'aspect musical pour cet article.

\section{1. La guitare de Claude Pavy}

20 Le guitariste français Claude Pavy à été l'un des premiers à introduire la guitare électrique dans le milieu de la musique contemporaine française. Commençant sa carrière comme musicien de variétés dans les studios d'enregistrement (où il prête ses services à Barbara ou Michel Legrand), il rentre dans la musique moderne par le hasard d'une rencontre avec le compositeur Tristan Murail. Il est bientôt fasciné par la musique contemporaine et devient le guitariste de l'ensemble de l'Itinéraire et un interprète de choix pour de nombreux compositeurs de renom comme Hugues Dufourt, ou Roger Tessier.

21 Le cas de Claude Pavy est un exemple de "déterritorialisation » : il a importé tout un vocabulaire de gestes instrumentaux et sonores de la musique de variétés dans la musique contemporaine, ce qui a permis aux compositeurs de réinventer ce vocabulaire, de lui donner un nouveau sens fonctionnel et sonore. Son travail a aussi été dans le sens d'une guitare électrique " globale ", où traitements et amplis sont aussi importants que la guitare elle-même. Finalement, d'une manière générale, sa collaboration avec les compositeurs dits «spectraux » a favorisé une esthétique sonore aérienne, de textures et de nappes, ce qui, pour la guitare électrique conçue en tant que système, signifie une dissociation entre l'entrée (in : le signal sonore de la guitare), et de la sortie (son sortant de l'ampli : des masses sonores soutenues, sans attaques ou traces de son "acoustique»). Autrement dit, son travail montre le début des possibilités sonores de la guitare électrique conçue en tant que réseau.

\section{2. La guitare électrique non amplifiée}

Je voudrais également souligner les possibilités de la guitare électrique non amplifiée. C'est-à-dire, la guitare électrique captée acoustiquement avec des micros. Le mélange entre le son amplifié de la guitare et le son de la guitare capté acoustiquement (que 
j'appelle son " pur ») donne à la guitare une palette dynamique et de timbre élargie. Il est difficile tracer les origines de cette guitare, mais cette façon de concevoir la sonorité de la guitare électrique commence à voir le jour dans les années 1990, dans le travail de studio de guitaristes de jazz comme Jim Hall ou Pat Metheny. Devenue une technique courante aujourd'hui, elle pourrait s'établir dans le jeu en temps réel grâce aux nouveaux systèmes de captation de cordes qui mélange micros et micros piezzo dans une même guitare. L'importance de cette façon d'approcher la guitare réside dans les possibilités esthétiques et fonctionnelles qu'elle suggère. Le son " pur » est pauvre en « hauteur » mais riche en timbre ; il fait émerger une nouvelle façon d'articuler les sons de la guitare, et met l'accent sur l'aspect bruitiste de l'objet physique, son côté métallique et un peu creux. Ces caractéristiques ajoutées au « corps » sonore produit pour l'ampli créent une dimension musicale peu explorée pour l'instant. Un bon exemple de ce type d'émergence est le travail du guitariste improvisateur anglais Derek Bailey, qui, travaillant avec une pédale de volume, construit un discours complexe entre son pur et amplifié.

\section{3. L'héritage du rock des années 1980}

Les années 1980, avec l'apogée du Heavy Metal et du guitariste rock virtuose, ont poussé l'aspect technique du jeu instrumental de la guitare électrique jusqu'au paroxysme. D'un point de vue musical, trois aspects constituent l'héritage significatif de cette époque : l'invention ou l'amélioration de modes de jeu de la guitare électrique, le développement des aspects techniques de la lutherie et de l'équipement environnant, et le développement d'un système de notation précis pour la guitare électrique.

La technique du tapping, popularisée par Eddie Van Halen et qui consiste à jouer avec les deux mains sur la touche, facilite l'agilité et la vitesse rapprochant la guitare d'instruments dotés d'une grande agilité mélodique comme la clarinette ou le saxophone. Appliquée d'une manière percussive, cette technique rapproche aussi la guitare du piano ou du clavecin. Au-delà du vocabulaire du rock, cette technique reste efficace pour la création de nuages sonores pointillistes à grande vitesse, à partir de champs harmoniques de plusieurs notes distribuées entre chaque main. L'apparition de nouvelles techniques issues du langage rock, a influencé la morphologie de la guitare électrique : développement de cordes extrêmement fines, modification du manche et $\mathrm{du}$ corps pour une meilleure ergonomie, développement d'une barre de vibrato flottante, etc. Ces innovations sont notées, étudiées, commentées, et surtout, mondialement divulguées dans plusieurs publications spécialisées (Guitar Player, Guitar World), et livres de transcriptions, qui développent un système de notation spécifique à la guitare électrique et réintroduisent la tablature comme moyen de représentation.

\section{4. Autres visions}

Grâce aux effets de delay, de reverb et de bouclage, la guitare peut être utilisée pour créer des textures sonores, des masses. Le travail de David Torn dans ce domaine reste exemplaire. La guitare peut être pensée "en dehors " d'elle-même, c'est-à-dire, s'éloignant d'une pensée instrumentale pour favoriser une vision conceptuelle de la guitare électrique en tant qu'outil global de création. Glenn Branca travaille avec la guitare électrique dans différents contextes, la traitant en tant qu'objet trouvé (et la 
modifiant de manière radicale), où la magnifiant en créant des grands ensembles. Elliott Sharp compose à partir de modèles physiques ou mathématiques externes à la guitare en utilisant les possibilités de traitement électronique, dont, Max/MSP. Keith Rowe explore les limites du « langage » sonore de la guitare électrique préparée.

\section{Création}

J'ai suggéré une idée générale de l'identité de la guitare électrique ainsi que quelques cas particuliers de domaines sonores et conceptuels émergents. Des questions se posent maintenant concernant la création: comment organiser ces domaines émergents ? Quoi faire du poids culturel de ces différentes identités de la GE ?

Je propose des réponses à ces questions à partir de mon travail personnel, qui consiste, pour l'instant, à manipuler, dans les domaines que je viens d'exposer, un réservoir de matériaux, et à organiser ce matériau d'une manière cohérente en dehors de la guitare. Il s'agit d'un travail de composition, dans le sens où je cherche à créer des formes sonores en utilisant l'outil "guitare électrique » et son bagage sonore du point de vue du compositeur, et en essayant de m'éloigner d'une vision de guitariste. Cette démarche soulève deux réflexions a priori.

Concernant les échelles temporelles, la plupart des traditions instrumentales de la guitare sont construites à partir du niveau meso, ou objet. En effet, les différents langages de la guitare électrique (jazz, rock, blues) se sont développés à partir d'une accumulation de durées locales adaptées à la mémoire et la perception des instrumentistes. Mon approche cherche, entre autres, à développer d'abord des formes globales, c'est-à-dire dans des échelles temporelles qui échappent à la perception immédiate du musicien, pour ensuite reconstruire les syntaxes locales. Si les cultures de la guitare électrique constituent pour la plupart des langages développés au niveau du vocabulaire et de la phrase, je voudrais réinventer ou modifier ces langages en construisant à partir des formes globales.

La réflexion sur les échelles temporelles suscite à son tour une réflexion sur le langage musical. Pour mes travaux, j'emprunte la définition d'André Boucourechliev de langage musical: «ensemble de lignes de force, des relations interactives entre ces forces et surtout de leur fonctionnement dans le temps ».

\section{1. Comment procéder?}

J'articule mon travail autour de quatre aspects :

- Ce que j'appelle un «modernisme de la guitare pure». Il s'agit de l'exploration des possibilités de la guitare électrique non-traitée, faisant, autant que possible, tabula rasa des vocabulaires « culturels » de la guitare (blues, rock, jazz). Trois approches principales (parmi d'autres possibles) :

- réorganisation non-tonale de groupes de hauteurs en utilisant différentes techniques compositionnelles de la musique contemporaine (sets, approche spectrale, fragmentation granulaire, etc.) ;

- travail sur une sonorité hybride guitare amplifiée/ guitare captée acoustiquement (renouvellement du répertoire d'articulation et de nuances) ;

- travail sur la guitare préparée. 
Cette approche s'inspire des visions compositionnelles des compositeurs comme Ligeti, par exemple. Le but de cette démarche est finalement la "création d'une syntaxe ", c'est-à-dire, la création d'un réseau organisé de relations entre matériaux musicaux qui fait émerger un langage musical cohérent et articulé.

- Travail sur les processus. Inspiré par le travail de compositeurs comme Steve Reich ou Tristan Murail, cette approche cherche à trouver des sources de processus (donc de forme) dans le réseau qui entoure la guitare électrique. Les répétitions d'une pédale de boucle peuvent être source de forme, ainsi que le spectrogramme de l'attaque d'une corde de guitare.

- Modélisation hors-temps, travail du global au local. Cette approche est inspirée de la pensée de Iannis Xenakis (Xenakis, 2006). Travailler avec des grandes échelles temporelles permet, par exemple, d'appliquer des modèles graphiques, mathématiques ou biologiques à la composition musicale.

- Pensée en réseau. Héritée de la pensée du compositeur Horacio Vaggione, cette approche consiste à construire un réseau d'outils (d'objets), au sein duquel un objet (une forme, une morphologie) sera manipulé et transformé, faisant émerger des événements singuliers, des nouvelles formes. Dans le domaine de la guitare, le réseau est constitué par l'instrument et les outils qui l'entourent (pédales, ordinateur, ampli) ; la composition implique une création d'un système cohérent, et donc la mise en relation des outils qui le composent. Cette approche permet de combiner tous les domaines de travail que j'ai cités avant.

L'avantage de l'unification des domaines de création dans un réseau de relations, est la possibilité d'appliquer un processus de pensée unifié (une idée compositionnelle) à un groupe d'approches hétérogène et fragmentaire. Cette approche globale confirme l'identité multiaxiale et modulaire de la guitare, dans le sens où elle met en valeur tous les composants du réseau : les pédales, l'ordinateur, l'ampli ne sont plus un «ajout » mais une partie intégrante de l'écosystème de la guitare électrique. Chaque outil du réseau est aussi source de forme, donc tous les membres du réseau jouent un rôle structurant dans la composition.

Mon approche est donc plurielle, et entend la composition comme une mise en relation de plusieurs domaines de création qui agissent sur des paramètres musicaux variés.

\section{2. Penser le réseau}

La guitare électrique est un instrument qui nécessite un moyen d'amplification pour devenir fonctionnel. Ceci nous pousse à penser la guitare électrique comme un tandem guitare-amplificateur et, comme un système complexe de production de son. Il s'agit d'un système (à priori) linéaire, qui dissocie la source génératrice du son (le geste instrumental créateur d'énergie : l'attaque des cordes) et le rendu sonore final (le son amplifié), ajoutant, sur le parcours du signal sonore, une série d'outils de contrôle qui sculptent le son. La guitare électrique est donc caractérisée par une scission entre source acoustique / rendu sonore amplifié, et par une multiplication de lieux de contrôle. Ceci fait d'elle un instrument radicalement différent de la guitare acoustique, pour laquelle il y a une continuité entre geste physique / son perçu.

Si l'on met l'accent sur le rôle actif que les outils de manipulation sonore entourant la guitare peuvent mener dans le processus créatif, on peut passer d'une idée de système linéaire simple (électrification d'une source acoustique), vers une idée de système complexe, en réseau, où l'identité sonore émerge des interactions entre source et outils de contrôle. Si la guitare électrique est depuis ses origines un système complexe où le 
signal sonore produit par les micros passe par une série d'outils de manipulation pour devenir « son " grâce à un amplificateur, aujourd'hui, les outils de création numérique faisant partie essentielle de ce système, la guitare électrique peut être pensée comme un instrument-réseau d'outils de création.

La guitare peut jouer trois rôles, dans son réseau (tel que je le conçois pour mon travail) :

- Source sonore en temps réel:

- simple : 1 canal (signal sortant de la guitare);

- complexe : plusieurs canaux (signal et captation acoustique de la guitare électrique en parallèle, plusieurs prises de son d'un ampli, sortie multicanal d'une guitare numérique, etc.).

- Source sonore hors-temps : la sonorité de la guitare électrique est utilisée comme matériau de base pour certains microréseaux comportant des processus de traitement en temps différé. Un exemple serait la création de nappes à l'aide d'une pédale de boucle. Le signal de la guitare ne devient message dans le réseau en temps réel, il sert seulement à créer une nouvelle source sonore dans le temps : la masse sonore créée par la boucle.

- Contrôleur : la guitare (son signal, le geste du guitariste, etc.) peut contrôler certains paramètres de fonctionnement du réseau. Un exemple serait les nuances du mode de jeu acoustique contrôlant la densité des masses granulaires produites pour le live électronique.

Il devient clair que l'informatique musicale est essentielle dans le réseau complexe qui constitue la guitare aujourd'hui, en particulier parce que, 1) elle brise la linéarité temporelle du réseau de base, donnant à la guitare plus de liberté dans son rôle de source hors-temps (avec les possibilités croissantes des ordinateurs et les techniques de traitement au niveau du microtemps, les processus linéaires d'enregistrement de source/reproduction se produisent à une vitesse suffisamment rapide pour être perçus par l'oreille comme temps réel; de cette manière, on peut jouer une note et, apparentement spontanément, générer une grande masse sonore à partir de fragments de la note initiale). 2) Elle brise, aussi grâce aux possibilités microtemporelles du live électronique, l'unité temporelle du réseau de base pour permettre la génération d'une pluralité de couches allant simultanément du niveau temporel méso (Roads) jusqu'au microtemps de la sonorité.

Les répercutions dans la création musicale de la création de ce modèle de guitareréseau sont variées. En lignes générales, on peut dire que la notion de composition se déplace vers la création d'un environnent d'émergence (le réseau), et le travail de création fragmentaire sur chacun des territoires qui comportent le système (écriture, programmation, improvisation, etc.). En somme, on a affaire à un espace de création pluriel qui n'est que la sublimation de l'idée de guitare dans toute sa complexité.

\section{3. Exemple pratique : « 5 Fragments pour guitare électrique »}

Ceci est un exemple de mon travail pour illustrer une forme de création en réseau. Pour « 5 Fragments pour guitare électrique », j'ai pensé mon réseau comme une mise en relation entre les domaines (territoires) de création suivants :

1. Le domaine de la composition musicale notée (avec des notes ou graphiques). Ceci est fondamentalement un travail loin de la guitare qui se concentre sur la prolifération de matériaux et la création dans une échelle temporelle macro (structure d'une pièce). Elle sert aussi, parfois, à « fixer » des trouvailles issues d'autres domaines du réseau. 
2. Le domaine "physique» du tandem guitare-joueur. Conçue comme un «lieu», donc un territoire visible où l'on trouve des choses, des "objets", la guitare est aussi source de matériaux et de forme (au niveau temporel meso, plus proche de la parole) ainsi que le corps et les gestes du musicien. Je conçois ce tandem comme une géographie sonore (la guitare), sur laquelle on peut agir physiquement (corps) pour trouver des « choses » (objets sonores). Pendant une performance, ce domaine ajoute une composante visuelle qui est aussi « composable».

3. Le domaine de l'improvisation. Ici, le musicien génère ou développe des formes en temps réel, se servant des outils à sa disposition. Dans mes pièces pour guitare, l'improvisation développe les processus et formes préétablies par l'écriture hors-guitare ; c'est-à-dire que la composition fournit le cadre global, les processus et les matériaux, et l'improvisation les développe et les articule dans le temps.

4. Le domaine des pédales. Soit sources de «coloration" (saturation), soit aussi sources de matériaux et formes via des traitements temporels (delays et boucles).

5. Le domaine du live électronique (Max/MSP). Lui aussi constitue un réseau composable. Source de polyphonie temporelle (microtemps), et de spatialisation (multiplication des sorties du réseau), il sert donc à complexifier la sonorité du réseau, ainsi qu'à la dynamiser (puisque il réagit aux actions du joueur).

40 Ces domaines, qui vont du conceptuel au purement pratique, touchent à des catégories qui se superposent. Cette multidimensionalité du réseau (où certains aspects se croisent, d'autres non) constitue la richesse de ce type de conception.

D’un point de vue technique, mon réseau est organisé de la manière suivante :

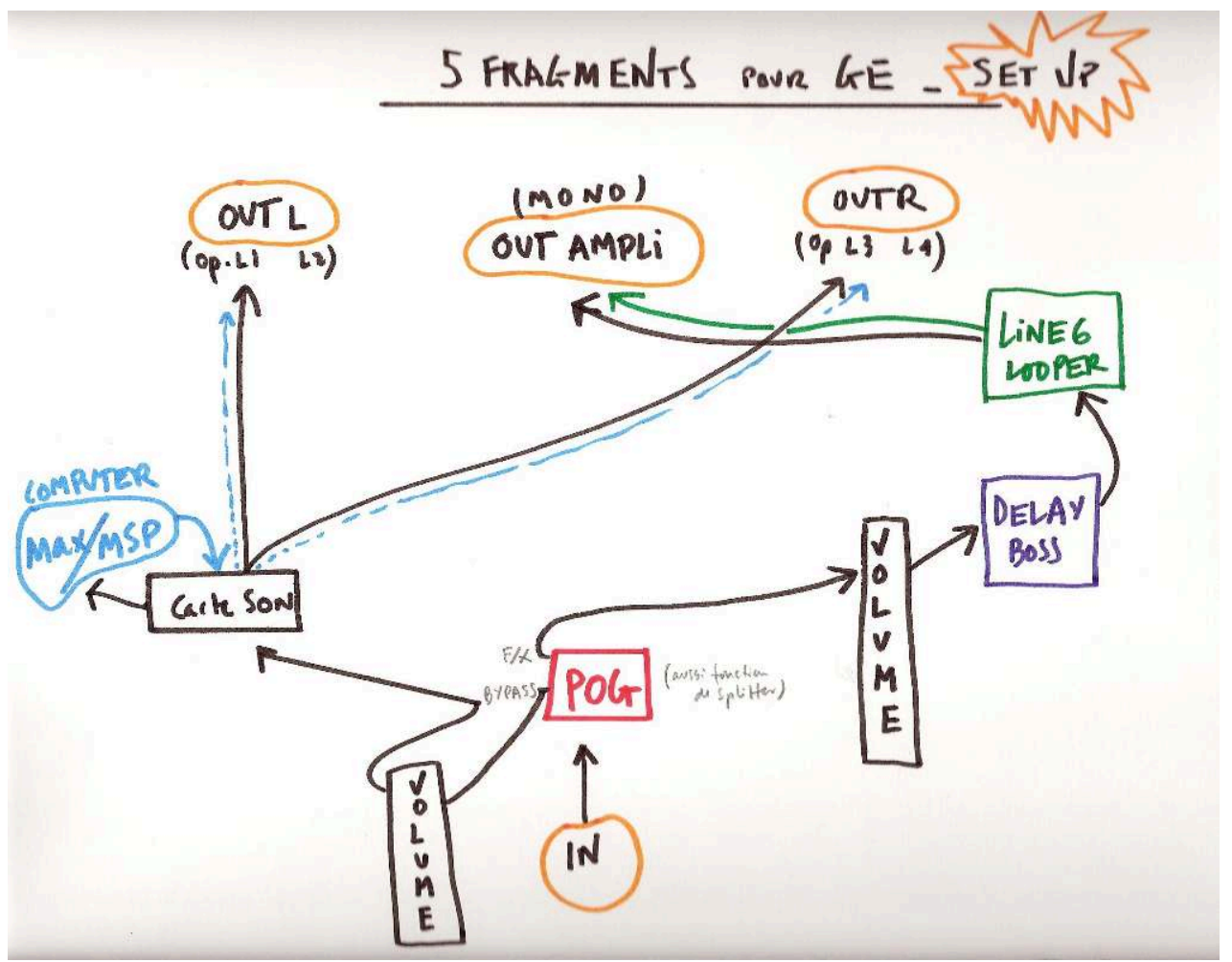

Ce système suggère plusieurs réseaux parallèles (signal, sortie pédale de boucle, captation acoustique, Max/MSP). Donc il s'agit d'un réseau de réseaux, tel que nous l'avons décrit auparavant. 

réseau, et des exemples sonores :

1. Concernant les matériaux:

a) Création de masses sonores

- Création de masses de type «spectral» (des accords issus du spectre d'un son) grâce a l'utilisation d'une pédale ElectroHarmonix POG, qui permet de baisser ou monter le son (hauteur) de la guitare d'une octave (donc élargissant la tessiture de la guitare de deux octaves, et donc permettant de générer des spectres assez riches), en combinaison avec une pédale de volume et de boucle (qui nous permettent d'enregistrer les différentes parties de la masse d'une manière séquentielle). Ex. 1 (liens Internet en bas de texte).

- Création de masses sonores de type granulaire, avec un comportement dynamique et spatialisé. Avec le logiciel Max/MSP j'ai construit des « patches » qui, à partir du signal de la guitare, génèrent des grains (entre 0 et 100 ms, environ). La taille des grains, la densité de la masse ainsi que son placement dans l'espace sonore stéréo sont définis par la vitesse et l'intensité des attaques à la guitare. Ex. 1

b) Création de « syntaxes » nouvelles, création de vocabulaire

- Relation entre la "géographie » de la guitare et le geste instrumental comme source de matériaux. Ici il s'agit de mettre en relation la prolifération écrite d'une cellule de hauteurs avec les cordes à vide, les différentes possibilités de geste instrumental liant les deux. Le résultat est le motif suivant :

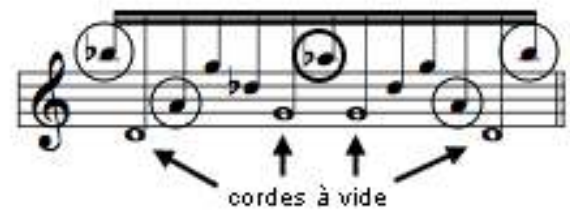

qui sera, ensuite développé par l'improvisation, avec un travail de traitement numérique en parallèle, censé développer ces nouveaux objets dans le domaine du timbre (effet doppler, mélange entre son propre et traité). Ex. 2

c) Application de nouvelles techniques de guitare pour générer des matériaux de sources variées :

- tapping générant des textures pointillistes. Ex. 5

- tapping comme extension harmonique d'accords.

d) Sonorités bruiteuses, non-harmoniques

- Travail avec la guitare préparée, manipulations avec des objets trouvés. Ex. 4 
e) Aspect théâtral, mouvements et gestes comme sources de forme

- Actionnement des pédales : bruit et geste intégrant la composition.

-Visualisation d'un processus : le 4 est organisé sur l'accumulation de bruitages qui se fait par l'application successive d'objets trouvés sur la guitare. Mettre un clip entre les cordes, frapper la guitare avec un bout de métal, ce sont des gestes significatifs qui deviennent des points de repère pour l'auditeur.

1. En ce qui concerne l'organisation de ces matériaux, je constate que :

50 a) mon réseau est une source complexe d'organisation puisque chaque domaine qui le compose génère des matériaux et des formes ;

51 b) donc, la composition pour réseau consiste à étudier les possibilités d'émergence du réseau et à les organiser d'une manière cohérente dans le temps, et dans une certaine esthétique.

Quelques possibilités que j'ai essayées de faire émerger :

- Le caractère formel de certains processus à l'intérieur du réseau : processus de création de masses spectrales audible dans l'ex. 1, processus d'accumulation, ex. 4.

- La possibilité de dissociation de rôles musicaux entre les différents domaines du réseau. Ceci permet de créer un espace sonore particulier au réseau, une sensation de perspective (contrepoint sonore). La dissociation entre masse sonore statique issue des pédales et objet sonore granulaire issu de l'ordinateur, par exemple, crée une sorte de contrepoint de masses assez expressif. Ex. 1, 5 et 3.

- La possibilité d'associer les différentes sonorités émergeant du réseau. Créant des plans sonores très proches, l'on peut créer une sorte de "feuilleté » sonore où l'attaque de la guitare est acoustique, le corps du son est numérique, et le decay vient de l'ampli et du son de la salle. Ses plans peuvent se combiner de façons différentes, donnant lieu à plusieurs variations sonores d'un thème. Ex. 2.

- Nouvelles formes d'improvisation. L'accumulation de contraintes (écrites), les possibilités de développement sonore (réseau), ainsi qu'une certaine volonté de m'approprier (en tant qu'improvisateur) des processus de répétition « machinale » issus de pédales, m’ont suggéré une façon de jouer basée sur la répétition et le contraste temporel entre cellules, ainsi que sur la différence entre champs sonores. Ex. 2

53 Les possibilités du réseau sont nombreuses, et parfois inattendues. Ce que je constate, après avoir travaillé sur cette pièce, c'est que cette conception réseau m'a permis de concilier plusieurs domaines de création dans lesquels, jusqu'à maintenant, je travaillais d'une façon isolée. La composition écrite, la pratique d'improvisateur et le travail sur l'électronique étaient des domaines qui coexistaient chez moi, qui essayent de se parler, mais qui, finalement, suivaient leur chemin indépendamment. Le pouvoir catalyseur de la guitare, ainsi que le fait d'accepter que cette multidimensionalité soit son essence, m'ont permis, avec l'aide de Max/MSP, de créer un domaine de travail fertile, dans lequel je me sens à l'aise, et dans lequel je peux faire «jouer» toutes mes envies créatives.

\section{Conclusion}

On peut penser la création musicale à partir d'une recherche sur des outils et des matériaux. Découvrir des matériaux et des outils implique comprendre la logique qui leur est propre et s'en servir pour les articuler dans des ensembles cohérents et 
expressifs. L'étude de la guitare électrique nous montre un instrument modulaire, pluriel, qui n'a fait qu'évoluer et changer depuis son apparition dans les années 1950. Vouloir réduire cet instrument à un modèle fixe serait ignorer une richesse qui lui est propre ; au contraire, accepter la pluralité de la guitare c'est s'ouvrir à une pensée complexe et fragmentaire qui reflète la vie de nos jours.

Mon travail essaie de mettre en valeur les domaines sonores dans lesquels la guitare électrique a développé une identité propre; les exemples de mes compositions montrent la volonté d'articuler et de mettre en relation différentes approches musicales et outils afin de faire émerger une musique nouvelle.

Les résultats de mes efforts viendront à leur tour alimenter de nouveaux réseaux dans lesquels, j'espère, l'identité de la guitare électrique ne cessera d'évoluer et de surprendre.

\section{BIBLIOGRAPHIE}

Bacon Tony (2001), The History of the American Guitar (from 1933 to present day), New York, Friedman/Fairfax Publishers, $112 \mathrm{p}$.

Baudrillard Jean (1990), La Transparence du Mal, Paris, Galilée, 180 p.

Bennet Andy, Dawe Kevin et al. (2001), Guitar Cultures, Oxford/New York, Berg, 215 p.

Bennet Andy (2001), “Introduction: Guitars, Cultures, People and Places”, in Guitar Cultures, p. 1-10.

Bennet Andy (2001), “Plug in and Play!: UK 'Indie-Guitar' Culture”, in Guitar Cultures, p. 1-10.

Brookes Tim (2005), Guitar. An American Life, New York, Grove Press, 340 p.

Boucourechliev André (1993), Le langage musical, Paris, Fayard, 186 p.

Dinnerstein Leonard, Nichols Roger, Reimers David (1996), Natives and Strangers. A Multicultural History of Americans, Oxford/New York, Oxford University Press, 369 p.

Evans David (2001), “The Guitar in the Blues Music of the Deep South”, in Guitar Cultures, p. 11-26.

Ligeti György (2001), Neuf essais sur la musique, Genève, Contrechamps, 215 p.

Linchestein Nelson et al. (2000), Who Built America? Working People and the Nation's Economy, Politics, Culture, and Society. Vol. 2, Since 1877, New York, Worth Publishers, 786 p.

Roads Curtis (2004), Microsound, Cambridge, MIT Press, 409 p.

Ruymar Lorene (1996), The Hawaiian Steel Guitars and its Great Hawaiian Musicians, Milwaukee, CenterStream Publications, Hal Leonard, 216 p.

Ryan John, Peterson Richard A. (2001), "The Guitar as an Artifact Icon: Identity Formation in the Babyboom Generation", in Guitar Cultures, p. 89-116.

Vaggione Horacio (1998), « L'espace composable. Sur quelques catégories opératoires dans la musique électroacoustique » in Espace : Musique/Philosophie, Paris, L'Harmattan, 1998, p. 152-166. 
Vaggione Horacio (2008), Composition musicale : représentations, granularités, émergences, in Intellectica, « Musique et Cognition », Anne Sédès (dir.), nº 48-49, p. 155-174.

Waksman Steve (2001), "Into the Arena: Edward Van Halen and the Cultural Contradictions of the Guitar Hero", in Guitar Cultures, p. 117-134.

Xenakis Iannis (2006), Musique de l'architecture, Marseille, Parenthèses, 443 p.

\section{Transcriptions}

Eddie Van Halen. Guitar Virtuoso, Van Nuys (USA), Alfred Publishing, 55 p.

1984. Van Halen (1991), Cherry Lane Music, Chester, NY (USA), 80 p.

Eat 'em and smile. David Lee Roth, Milwaukee, WI (USA), Hal Leonard, 104 p.

\section{Liens Internet vers les exemples sonores}

Ex. 1 : http://cicm.mshparisnord.org/media/ExtraitFRG1.mp3

Ex. 2 : http://cicm.mshparisnord.org/media/ExtraitFRG2.mp3

Ex. 3 : http://cicm.mshparisnord.org/media/ExtraitFRG3.mp3

Ex. 4 : http://cicm.mshparisnord.org/media/ExtraitFRG4.mp3

Ex. 5 : http://cicm.mshparisnord.org/media/ExtraitFRG5.mp3

\section{INDEX}

Mots-clés : composition, guitare électrique, réseau

\section{AUTEUR}

\section{SANTIAGO QUINTANS}

Coordinateur du programme Jazz au conservatoire (CRD) du Mans, compositeur. Doctorant au CICM université Paris 8 